\title{
Analyzing the Perception of Online Sheet Music Search: A Case Study
}

Dinuka De Silva, Saman Hettiarachchi

Department of Computer Science

Informatics Institute of Technology Colomo

Sri Lanka

dinuka.2013513@iit.ac.lk,saman.h@iit.ac.lk

ABSTRACT: Among the number of formats of representing music, music sheets carrying a significant importance even though sheet music is not used by everyone. For musicians, composers and for those who are practicing sheet music, that is more convenient and the best materials as well. As defined by western musicians, it has grown and now widely available even in the internet. As it become wider, the need of navigation and searching has become required because of the difficulties in browsing large amount of data. Online searching approaches different ways of querying such as text, audio etc. This paper is an investigation of the perception towards searching sheet music online in general. This includes a brief analysis of user needs and expectations towards the perception of online sheet music search contains an online survey, expert interviews and a discussion outlining the existing approaches and future work needed.

Keywords: Music, Information Retrieval, Music Information Retrieval, Sheet Music, Online Search

Received: 12 May 2018, Revised 10 June 2018, Accepted 16 June 2018

DOI: $10.6025 / \mathrm{jcl} / 2018 / 9 / 3 / 99-105$

C 2018 DLINE. All Rights Reserved

\section{Introduction}

This online data in increasingly wider day by day. The complexity of navigating towards required data online is becoming complicated and time consuming. As a result, the development of search capabilities is a continuation. Out of that, music data carrying an its own complexity because of its data formats and structure. Music data could be represented in many formats. Audio's of music could be the mostly available and easily producible music format. And then videos could be the most widely using format for representing a music recording. Sheet music could be the most standard way of representing a music which is not used by everyone but widely used by musicians, music experts and those who are knowledgeable in music. All kinds of music formats are available in the internet, the challenge here is navigating towards the relevancies, the limitations and improvements needed for existing solutions.

Sheet music could be even not known to many people. But, for those who can understand sheet music, it could be the best requirement. Even when it's needed to find a required music, the requirement could be a music sheet rather audios or videos. And

International Journal of Computational Linguistics Research Volume 9 Number 3 September 2018 
perhaps, there couldn't have a certain way of expressing the expectation of the requirements. This case study focuses on analyzing the perception of online sheet music search among general to understand what are real user expectations and existing nature.

\section{Methodology}

An online survey was published to get general feedbacks. A snow ball sampling [1] methodology was followed to collect the responses by encouraging the network to assist to get further responses. The survey was distributed among a diverse audience to get the feedbacks from individuals with different skills and cultures without looking at music preferences or knowledge. The questions were carefully defined in a way to capture the responders point of view in online sheet music search and its importance to both the responder and all over. And also the survey was directly distributed to some of the musicians and researchers to get their feedback and advice.

A set of interviews were conducted aiming to brainstorm with their knowledge. It was mostly focused on capturing the real requirements of musicians and existing drawbacks to carefully understand the current state of the approach and needs towards online sheet music search.

\section{Background}

Information standardization and retrieval is a real need not only for music. It's a broad topic that has taken the attention of scientist and researchers all over. The online information is increased day by day. As a result, it has opened up the need of browsing information effectively and efficiently.

Music could be known and unknown as well. Sometimes, the melody could be familiar thought the song or its title could be not known. In that sense it has some information, but cannot convert that into a textual representation [2] which could make a barrier to search it online which has been even further justified by previous studies [2].

Perhaps, music could be considered as another language [3], [4]. A single melody could be called a monophonic sequence of music which could be mostly considered to have native linguistic patterns. And a melody consists of harmony parts could be called as a polyphonic music [5], [6] which is where the music goes beyond native linguistic patterns which open up the complexity towards the effective information retrieval.

Music requirements could be not just pitches. But also rhythm which is still complex to be converted to a textual representation which has also been addressed by many researchers [7] and shown their importance towards areas such as online education, authentications.

Overall, sheet music could be not a general requirement for everyone. Based on who it's applied to which task it could be beneficial or not. At the same time, it must require a knowledge, a practice and more importantly an interest.

\section{Results}

\subsection{Online Survey}

The survey was built using google forms and distributed via emails, social media, contacts and the contacts network. Also it was distributed to some of the music research email groups to get the attention of music and research experts around the world. The questionnaire was built with following question.

\section{1) Searching Music}
a) How
b) How often
c) Why and importance

2) Knowledge about Sheet Music

3) Searching Sheet Music

$100 \quad$ International Journal of Computational Linguistics Research Volume 9 Number 3 September 2018 

a) How
b) Features and their importance

4) Importance of Searching Sheet Music
a) Opinions
b) Recommended Stakeholders
c) Non-functional features

5) Conclusion
a) Contact and Biographical Details
b) Comments and feedbacks

From the 192 responses received to the date for about last two months, it had been distributed to 33 countries. Also it's interesting to see that about $20 \%$ of the responders are reluctant to mention their country. But, more than $50 \%$ of the responders are really interested in the research and given the contacts to be notified about the results of the study. that shows the interest and more work is expected in the area of searching music and sheet music.

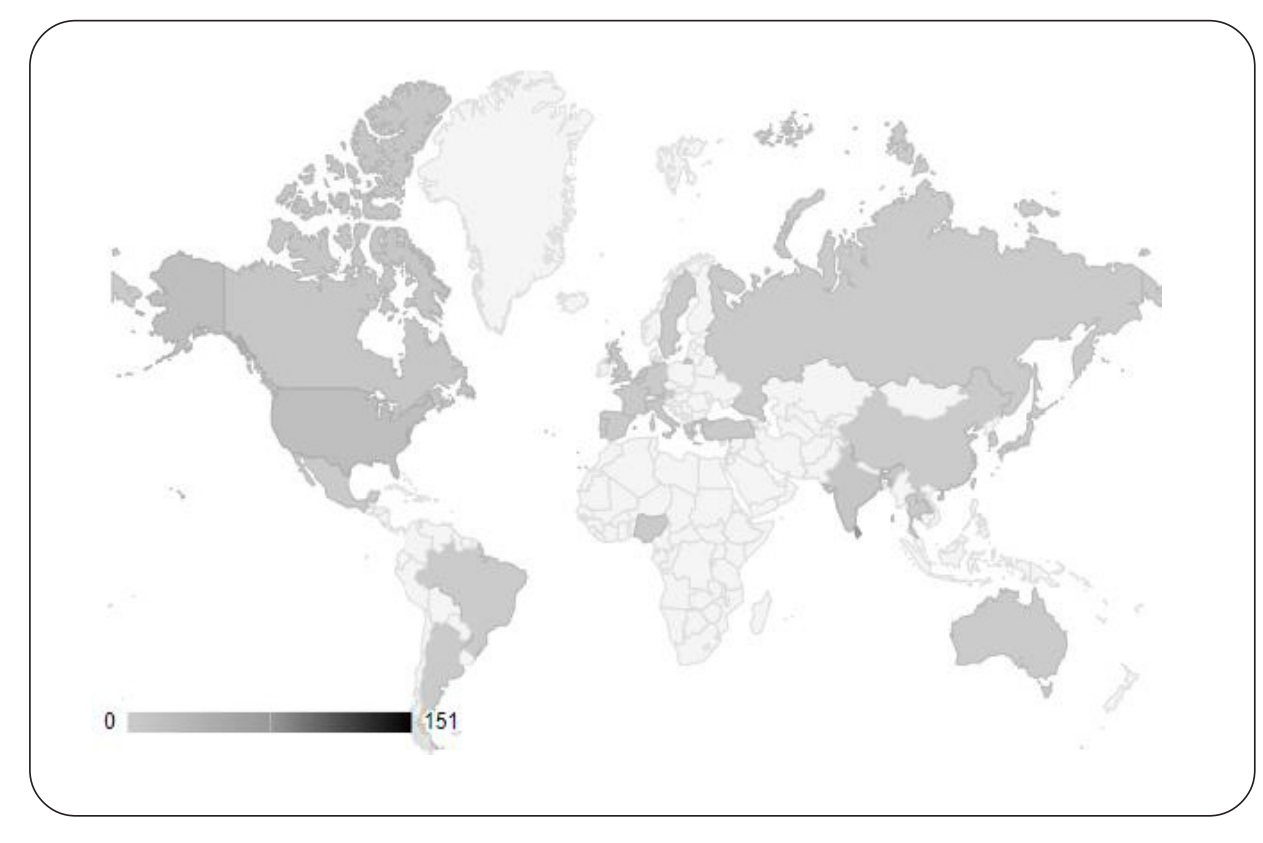

Figure 1. Diversity of the Survey Responses

It's seen that searching music is a very frequent task for everyone. From the responses, more than $95 \%$ of the responses says it is. More than $80 \%$ of the responders says that they do searching music at least few times a week. Also, their feedback says that, it's to find music mostly for entertainment and relaxation. In overall, YouTube, Google and Spotify has been the mostly used online search engines for searching music. Also, some of the responders are used to not popular search engines as well. In terms of searching capabilities, more than $95 \%$ has found text based searching and around $30 \%$ of them has seen audio and sound based searching. And more importantly, only $5 \%$ of the responders has at least seen some of the applications that supports sheet music based search engines which shows that less work is available in that area. Also it clearly gives another proof that sheet music based search could be not a general need.

Among the mixed background of these responders, it shows that, most of them know what sheet music although they are not knowledgeable. And with an explanation of what sheet music is given by an explanation extracted from Wikipedia, sheet music is not an unknown word to more than $80 \%$ of the responders. But, only $40 \%$ of them are really understand what sheet music is. And actually those who understand and has an interest in sheet music are really looking for searching sheet music. But, currently available capabilities have become more limited to text based searching when musicians are mostly looking for audio and sheet music based search capabilities. But, in overall the text base search is more prioritized and everything else comes only after that 
based on the user feedbacks.

Those who are interested in searching sheet music, has mostly gone to MuseScore [8], MusiPedia [9] and IMSLP [10]. Both MuseScore and IMSLP has only text based searching and IMSLP has hypertext searching additionally. Different to them, MusiPedia has the capabilities of searching by a music which could be a melody or a rhythm which has gone step further towards the interest of musicians over text based searching.

Out of those who aware what is sheet music is, the capabilities looking were analyzed by giving a Likert Scaling with four options to indicate the perception towards five mentioned capabilities Hum, Text, Audio, Video, Sheet Music and Other. when comparing the needs looking for, it shows that still text based search is essential because that's the simplest way to search a music quickly and all the other alternative are needed only for unknown searches and depth searching which is mostly required only to Musicians or Researchers. And importantly, there is a need of sheet music and audio based search which most of the musicians are looking for. When looking at those who are really working with music and music related researchers, sheet music based search has taken the most priority because of its less availability. Overall, video and hum based searching off course has taken an interest among the musicians as same as sheet music. And Sheet Music based searching capability has taken a moderate priority. But, for than $20 \%$ of the responders, it's an importance feature to have while more than $70 \%$ of overall assume it could be.

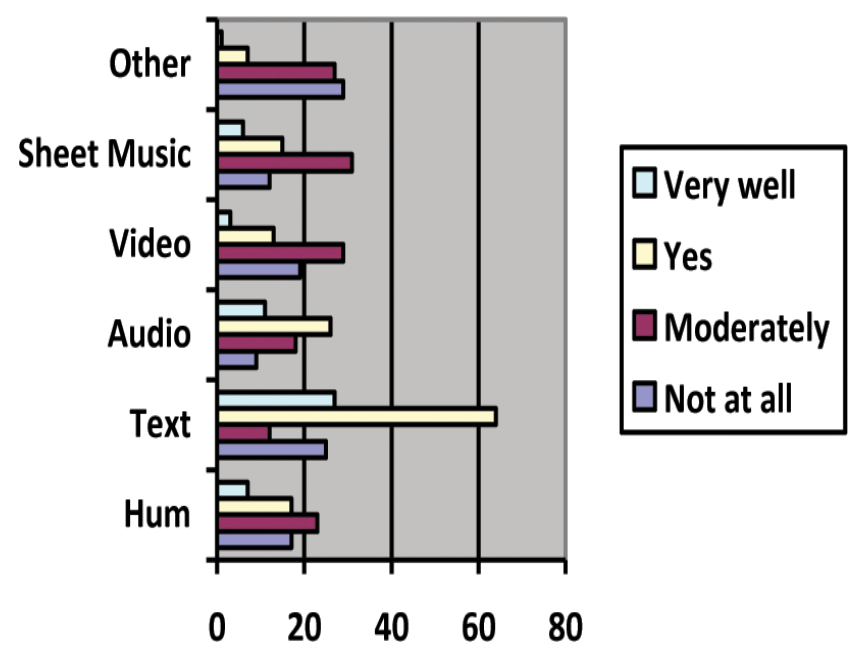

Figure 2. Importance of Searching Query Capabilities for Music

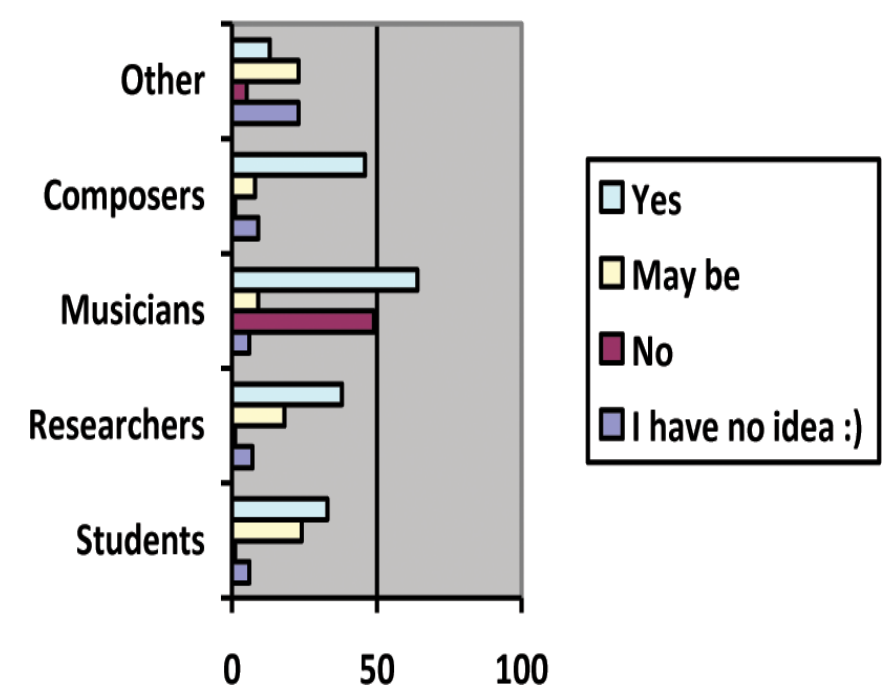

Figure 3. Stakeholder Recommendations to Searching Sheet Music 
To get a general feedback on to whom the searching sheet music be important to, a question with a Likert Scaling was published with four scaling points describing the importance level based on the responder's point of view. The questions were also categorized based on different stakeholders. The recommendations given states that it could be an essential to Musicians and Composers which shows that general users don't see an importance in sheet music and seeing much interest in other types of media. Also, many responders has identified that students and researchers [11] also could benefit out of browsing sheet music which shows the academic focus towards sheet music.

A verbal feedback taken on the importance of sheet music from the responders, clearly shows that sheet music is more irrelevant to general because that needs knowledge of sheet music and more importantly an interest towards. But, when carefully looking at the responses from researchers, musicians and those who are knowledgeable in music, a vast interest is seen towards searching sheet music and given the interest to be updated for further investigations as well. Some of the importance mentioned were browsing an arrangement by melody or unknown music. And then finding different editions of the same music that could be in different keys or time signatures which could represent the same music arrangement. But also, to explore different variations of compositions off course the responders have shown an interest.

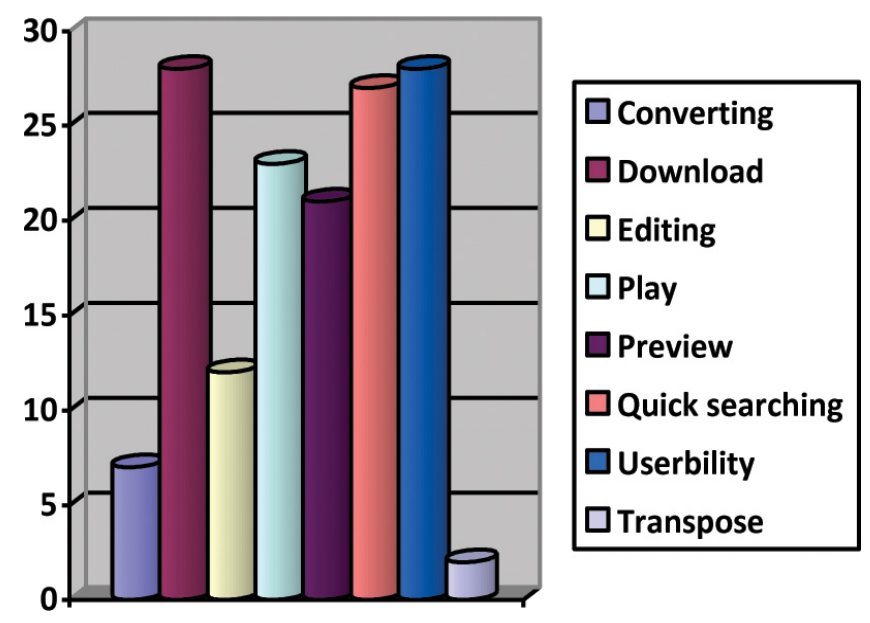

Figure 4. Importance Of Non-functional Features of a Sheet Music Search Engine

Non-functional requirements are also important to get the visibility among competitors. And that's where the users feel about any software. So, a question was published about the non-functional features looking in a search engine for sheet music with few specified answers to elaborate the user a bit more on the question. The responses show that, the usability of the software and easy downloads are things many users are looking for. And the efficiency of searching has been identified by many responders as a requirement. The online availability of editing and previewing of either sheet music or audio could be a new feature which has seen by many responders as needed. Also, some of them has needed transpositions and conversions between keys and formats respectively.

\subsection{Interviews}

A set of interviews were conducted with few experts who has some background in music and computer science.

While the individual discussion with first interviewer, it's was mostly understood that even some of the musicians are not specifically using sheet music but, some alternatives such as Tablature. His band consist of two guitarists, one mandolin player and drums. They are doing concerts based in their city and mostly playing country rock music. So, some, the songs are found from internet and some are just by hearing. Whenever a song or any music is unknown they've been looking for a solution may be to play it in the guitar and find exact chords or lyrics.

The second interviewer was mostly an engineering expert and having knowledge about music. In his work he has seen the need of grouping and measuring the similarities of sheet music for the use of marking student assignments efficiently. And then, he has seen a gap in this area which is because of the complexity of polyphonic structure of music and the nature of music which has a 
more context than how it's seen.

\begin{tabular}{|l|l|}
\hline Interviewer & Background \\
\hline 1 & $\begin{array}{l}\text { A literature major having product management carrier for more than 17 years. } \\
\text { A guitarist and a musician having their own band based in US. }\end{array}$ \\
\hline 2 & $\begin{array}{l}\text { A computer science major working as a software engineering manager. } \\
\text { Having a background in music and currently following his master's degree in University of Oxford. }\end{array}$ \\
\hline 3 & $\begin{array}{l}\text { A seneor lecturer having a PhD in Natural Language Processing. } \\
\text { His research interests are in Computational Linguistics, Machine Translation, Machine Learning, } \\
\text { Intelligent Systems, Bioinformatics. And also he has some background and knowledge in sheet music. }\end{array}$ \\
\hline
\end{tabular}

Table 1. Interviewers

The third interviewer is from academic who is working mostly with students and research. Some of the areas identified needed work while the discussion was the analysis of harmony parts which really need more work.

In overall with all three interviews, it was notices that analysis of polyphonic structure of music has a knowledge gap which is still pending. And another format of music commonly used was identified which is Tablature mostly used for guitar music. The identification of unknown music is something musicians are looking for. And similarity matrices of music have a need even for effective education.

\section{Discussion}

Sheet music is a well-structured format for representing music. And audios and videos could be not structured as sheet music. But, Sheet music is not a general need. Even for those who are knowledgeable in sheet music that's not the most suitable format. Many are interested in audios and videos. For practicing sheet music, it required both the knowledge and an interest.

But, for musicians, composers and for those who are practicing sheet music, sheet music is the best requirement. When looking at available sheet music search engines, most are text based search engines. Still the text searching is required even for sheet music because that's the quickest way of searching anything. And moderately, there is a need of searching for rhythms, unknown melodies, different editions, arrangements etc. Then the need of searching by sheet music comes which somewhat exists even now though not much popular. Musipedia [9] is one of them which provides monophonic melody and rhythm searching capabilities.

With observations from the study, it states that, more work is needed to improve the searching capabilities of online sheet music. Analysis of polyphonic music is an area need lot more work.

\section{Conclusion}

The study reveals the importance of the availability of searching sheet music online and the required additional features outlining their importance to musicians, composers and for those who practice sheet music. Searching by sheet music has been identified as a required featured that doesn't exist as expected to the date. And the discussion has clearly states the knowledge gap that could be addressed by further research.

\section{Acknowledgment}

This study is a part of an undergraduate research project conducted by Informatics Institute of Technology affiliated with University of Westminster. This is to acknowledge the guidance and support given for conducting this research. And also, highly appreciate the valuable time of the responders who attended the surveys and interviews.

104 International Journal of Computational Linguistics Research Volume 9 Number 3 September 2018 


\section{References}

[1] Goodman, L. A. Snowball Sampling, The Annals of Mathematical Statistics, 32. Institute of Mathematical Statistics, 148-170.

[2]Lee, J. H. (2010). Analysis of user needs and information features in natural language queries seeking music information, J. Am. Soc. Inf. Sci. Technol., 61 (5) 1025-1045, May.

[3] Cooke, D. (1959). The Language of Music. Oxford University Press, 1959.

[4] Hudson, W. C. (1973). Music: A physiologic language., J. Music Ther., 10 (3) 137-140, 1973.

[5] Ning Hu, R. B. Dannenberg., Tzanetakis, G. (2003). Polyphonic audio matching and alignment for music retrieval, In: 2003 IEEE Workshop on Applications of Signal Processing to Audio and Acoustics (IEEE Cat. No.03TH8684) 185-188.

[6] Lemström., Tarhio, K. J. (2000). Searching monophonic patterns within polyphonic sources, Content-Based Multimedia Information Access - 2. LE CENTRE DE HAUTES ETUDES INTERNATIONALES D'INFORMATIQUE DOCUMENTAIRE, 12611279.

[7] Peters, G., Cukierman, D., Anthony, C., Schwartz, M. (2006). Online Music Search by Tapping, Springer, Berlin, Heidelberg, 178-197.

[8] Sheet music | MuseScore. [Online]. Available: https://musescore.com/. [Accessed: 02-Jun-2018].

[9] Irwin, K. (2008). Musipedia: The open music encyclopedia, Ref. Rev., 22 (4) 45-46.

[10] Guo, E. W. (2006). IMSLP/Petrucci Music Library, IMSLP/Petrucci Music Libr., 16.

[11] Thomas, V., Fremerey, C. , M. M.-D. F., and undefined 2012, Linking sheet music and audio-Challenges and new approaches, drops.dagstuhl.de. 